\title{
Susceptibility of rabbits to SARS-CoV-2
}

2 Authors: Anna Z. Mykytyn, Mart M. Lamers, Nisreen M.A. Okba, Tim I. Breugem, Debby

3 Schipper, Petra B. van den Doel, Peter van Run, Geert van Amerongen, Leon de Waal, Marion

4 P.G. Koopmans, Koert J. Stittelaar, Judith M.A. van den Brand, Bart L. Haagmans

\section{Affiliations:}

7 Erasmus Medical Center, Rotterdam, the Netherlands (A.Z. Mykytyn, M.M. Lamers, N.M.A.

8 Okba, T.I. Breugem, D. Schipper, P.B. van den Doel, P. van Run, M.P.G. Koopmans, B.L.

9 Haagmans)

10 Utrecht University, Utrecht, the Netherlands (J.M.A. van den Brand)

11 Viroclinics Biosciences B.V., Viroclinics Xplore, Schaijk, the Netherlands (G. van Amerongen, 12 L. de Waal, K.J. Stittelaar). 


\section{Abstract}

21 Transmission of severe acute respiratory coronavirus-2 (SARS-CoV-2) between livestock and

22 humans is a potential public health concern. We demonstrate the susceptibility of rabbits to

23 SARS-CoV-2, which excrete infectious virus from the nose and throat upon experimental

24 inoculation. Therefore, investigations on the presence of SARS-CoV-2 in farmed rabbits should

25 be considered.

\section{Text}

Severe acute respiratory syndrome coronavirus 2 (SARS-CoV-2) caused a pandemic only months after its discovery in December 2019 (1). Slowing down its spread requires a full understanding of transmission routes, including those from humans to animals and vice versa. In experimental settings, non-human primates, ferrets, cats, dogs and hamsters have been found to be susceptible to SARS-CoV-2 infection (2-4). Moreover, ferrets, cats and hamsters were able to transmit the virus via the air $(2,4,5)$. In domestic settings, both dogs and cats have been found to carry the virus, displaying very mild to more severe symptoms, respectively (5). Recently, SARS-CoV-2 has been isolated from mink at multiple Dutch farms. Workers at those farms carried viruses that were highly similar to the viruses detected in mink and phylogenetic analyses

37 supported transmission from mink to workers (6). Thus, measures to control the spread of SARS-

38 CoV-2 should also include preventing spill over into potential reservoirs, especially since

39 infectious agents can spread rapidly in livestock due to the high densities at which some animals

40 are kept. Given the fact that rabbits are commonly farmed worldwide, we investigated the

41 susceptibility of rabbits to SARS-CoV-2. 
The study

Angiotensin converting enzyme 2 (ACE2) dictates the host range for SARS

47 fusion of the viral and host membranes (8). Contact residues of human and rabbit ACE2 critical

48 for binding S are relatively well conserved (9). We overexpressed ACE2 from different species

50 infection. Successful infection of both SARS-CoV-2 pseudovirus and authentic virus was

51 observed for human, rabbit and the Chinese horseshoe bat (Rhinolophus sinicus) ACE2 (Figure

52 1, panel A and B). The ACE2 from a distantly related bat, the great Himalayan leaf-nosed bat

53 (Hipposideros armiger), did not support infection and served as a negative control. Transfection

54 of rabbit ACE2 rendered cells susceptible to SARS-CoV-2 infection demonstrated by a clear

55 overlap between infection and ACE2 expression (Figure 1, panel C).

Next, we inoculated three rabbits with $10^{6}$ tissue culture infectious dose 50 (TCID $\left.{ }_{50}\right)$

57 SARS-CoV-2 for a 21 day follow up. None of the inoculated animals showed clinical signs of

58 infection. As shown in Figure 2 (panel A), we found viral RNA in the nose for at least twenty-

59 one days (mean shedding of 15.33 days, $\mathrm{SD}=5.13$ ), up to fourteen days in the throat (mean

60 shedding of 11.33 days, $\mathrm{SD}=2.52$ ) and up to nine days in the rectum (mean shedding of 5 days,

$61 \mathrm{SD}=3.61$ ). Infectious virus shedding from the nose lasted up to seven days (mean shedding of

626.67 days, $\mathrm{SD}=0.58$ ) with a peak at day two, followed by a second peak at day seven post

63 inoculation (p.i.) (Figure 2, panel B). In the throat, infectious virus was detected only on day one 
64 p.i. for one animal. No infectious virus was detected in rectal swabs. All animals followed up to

65 day 21 seroconverted with plaque reduction neutralization test $\left(\mathrm{PRNT}_{50}\right)$ titers of 1:40, 1:320 and

$66 \quad 1: 640$.

67 Additionally, three groups of three animals were inoculated with either $10^{4}, 10^{5}$ or $10^{6} \mathrm{TCID}_{50}$

68 SARS-CoV-2 and swabs were taken for four days before the animals were euthanized and

69 autopsied. All animals inoculated with $10^{6} \mathrm{TCID}_{50}$ were viral RNA positive in the nose and

70 throat for at least four days with a single animal positive in the rectum at day three (Figure 2,

71 panel C). Animals inoculated with $10^{5} \mathrm{TCID}_{50}$ were RNA positive in the nose for at least four

72 days, for at least three days in the throat but not in the rectum (Figure 2, panel D). These animals

73 also shed infectious virus in the nose for up to three days (mean shedding of 1.67 days, SD=1.53)

74 and one animal shed virus two days post infection in the throat (data not shown). Animals

75 inoculated with $10^{4} \mathrm{TCID}_{50}$ did not shed any detectable viral RNA (Figure 2, panel E). Although

76 nasal turbinates yielded on average $8.42 \times 10^{3}$ RNA copies $/ \mathrm{ml}$, lung homogenates of animals

77 inoculated with $10^{6} \mathrm{TCID}_{50}$ virus were found viral RNA negative (Figure 2, panel F). Despite the

78 fact that no viral RNA was detected in the lungs, histological examination of the lungs of

79 infected animals sacrificed four days p.i. revealed a multifocal mild to moderate increase in

80 alveolar macrophages in the alveolar lumina with multifocal presence of few neutrophils. Mainly

81 associated with the terminal bronchioles, multifocal mild thickening of the septa, with

82 infiltrations of neutrophils, eosinophils and occasional lymphocytes, plasma cells and

83 macrophages was observed (Figure 2, panel G). Mild multifocal necrosis of alveolar epithelial

84 cells and the presence of a few enlarged, syncytial cells in the alveolar lumina were seen (Figure

85 2, panel $\mathrm{H}$ ). There was mild peribronchiolar and peribronchial lymphoplasmacytic infiltration

86 with eosinophils and moderate to severe bronchus-associated lymphoid tissue proliferation 
87 (Supplementary figure 1, panel A). Some animals showed enlarged tracheo-bronchial lymph

88 nodes consistent with mild lymphoid hyperplasia. In the nose, there was multifocal infiltration of

89 moderate numbers of eosinophils and lymphoplasmacytic infiltrates in the olfactory epithelium

90 (exocytosis) and in the lamina propria, alongside mild hyperplasia and hypertrophy of the

91 olfactory epithelium (Supplementary figure 1, panel B). Mild eosinophilic exocytosis was

92 present in the trachea.

\section{Conclusions}

This study demonstrates that rabbits are susceptible to SARS-CoV-2. While the infection

95 is asymptomatic, infectious virus with peak titers corresponding to $\sim 10^{3} \mathrm{TCID}_{50}$ could be

96 detected up to day seven post inoculation in the nose. The minimum dose to establish productive

97 infection was $10^{5} \mathrm{TCID}_{50}$, indicating that virus transmission between rabbits may be less

98 efficient compared to ferrets and hamsters. The use of young, immunocompetent, and healthy

99 New Zealand White rabbits in this study however may not reflect virus shedding and disease in

100 other rabbit breeds or rabbits at different ages. Thus, surveillance studies - including serological

101 testing - may be needed to assess the presence of SARS-CoV-2 in farmed rabbits.

Viral shedding in rabbits occurred in a biphasic pattern, which was also observed for innate immune responses that act within days, followed by adaptive responses that generally take one week to be activated. These observations are in line with recent findings that the presence of neutralizing serum antibodies in humans negatively correlates with infectious virus shedding,

107 and that shedding of viral RNA outlasts shedding of infectious virus (11). The presence of eosinophils in the nose and lungs of infected animals suggests a possible helper T cell 2 (Th2)mediated immune response. The preferential upper respiratory tract infection in the absence of 
110 robust replication in the lower respiratory tract of rabbits resembles what has been observed in

111 experimentally inoculated ferrets (5).

The transmission of SARS-CoV-2 to mink caused viral spread between farm animals

113 and spillover to humans, resulting in mass culling of mink to limit the spread of the virus (6).

114 This study provides evidence of susceptibility of rabbits to SARS-CoV-2 infection warranting

115 further investigations on the presence of SARS-CoV-2 in farmed rabbits.

117 Acknowledgments

This research is partly financed by the Netherlands Organization for Health Research and

121 Sciences \& Health, to stimulate public-private partnerships.

\section{Author Bio}

124 Anna Mykytyn is a PhD candidate at Erasmus Medical Centre, Rotterdam. Her research interests

125 are the pathogenesis and transmission of coronaviruses including SARS-CoV-2. 


\section{References}

131 1. World Health Organization. Coronavirus disease (COVID-19) pandemic. 2020 [cited;

132 Available from: https://www.who.int/emergencies/diseases/novel-coronavirus-2019

133 2. Chan JF, Zhang AJ, Yuan S, Poon VK, Chan CC, Lee AC, et al. Simulation of the

134 clinical and pathological manifestations of Coronavirus Disease 2019 (COVID-19) in golden

135 Syrian hamster model: implications for disease pathogenesis and transmissibility. Clin Infect

136 Dis. 2020 Mar 26.

137 3. Rockx B, Kuiken T, Herfst S, Bestebroer T, Lamers MM, Oude Munnink BB, et al.

138 Comparative pathogenesis of COVID-19, MERS, and SARS in a nonhuman primate model.

139 Science. 2020 May 29;368(6494):1012-5.

140 4. Sia SF, Yan LM, Chin AWH, Fung K, Choy KT, Wong AYL, et al. Pathogenesis and

141 transmission of SARS-CoV-2 in golden hamsters. Nature. 2020 May 14.

142 5. Shi J, Wen Z, Zhong G, Yang H, Wang C, Huang B, et al. Susceptibility of ferrets, cats,

143 dogs, and other domesticated animals to SARS-coronavirus 2. Science. 2020:eabb7015.

144 6. Oreshkova N, Molenaar R-J, Vreman S, Harders F, Munnink BBO, Hakze R, et al.

145 SARS-CoV2 infection in farmed mink, Netherlands, April 2020. bioRxiv.

$146 \quad 2020: 2020.05 .18 .101493$.

147 7. Zhou P, Yang XL, Wang XG, Hu B, Zhang L, Zhang W, et al. A pneumonia outbreak

148 associated with a new coronavirus of probable bat origin. Nature. 2020 Mar;579(7798):270-3.

149 8. Li F. Structure, Function, and Evolution of Coronavirus Spike Proteins. Annu Rev Virol. 1502016 Sep 29;3(1):237-61.

151 9. Zhao X, Chen D, Szabla R, Zheng M, Li G, Du P, et al. Broad and differential animal 152 ACE2 receptor usage by SARS-CoV-2. J Virol. 2020 Jul 13. 
153 10. Hartman AL, Nambulli S, McMillen CM, White AG, Tilston-Lunel NL, Albe JR, et al.

154 SARS-CoV-2 infection of African green monkeys results in mild respiratory disease discernible

155 by PET/CT imaging and prolonged shedding of infectious virus from both respiratory and

156 gastrointestinal tracts. bioRxiv. 2020:2020.06.20.137687.

157 11. van Kampen JJA, van de Vijver DAMC, Fraaij PLA, Haagmans BL, Lamers MM, Okba

158 N, et al. Shedding of infectious virus in hospitalized patients with coronavirus disease-2019

159 (COVID-19): duration and key determinants. medRxiv. 2020:2020.06.08.20125310. 
bioRxiv preprint doi: https://doi.org/10.1101/2020.08.27.263988; this version posted August 27, 2020. The copyright holder for this preprint (which was not certified by peer review) is the author/funder, who has granted bioRxiv a license to display the preprint in perpetuity. It is made available under aCC-BY-NC-ND 4.0 International license.

173

174

175

176

177

178

179

180

181

182

183
A

\section{SARS-CoV-2 pseudovirus}
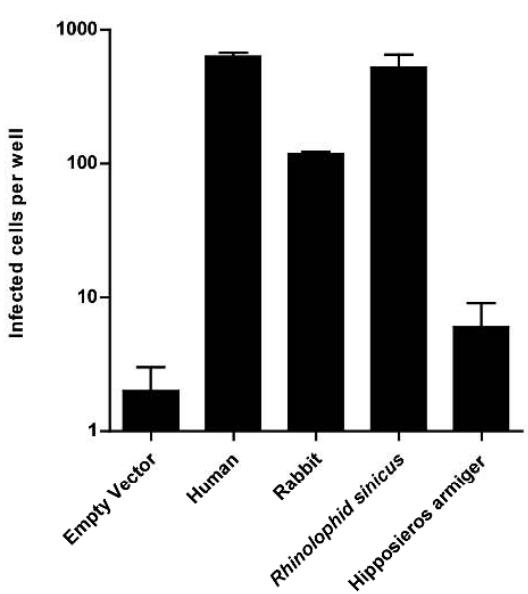

B

SARS-CoV-2 live virus

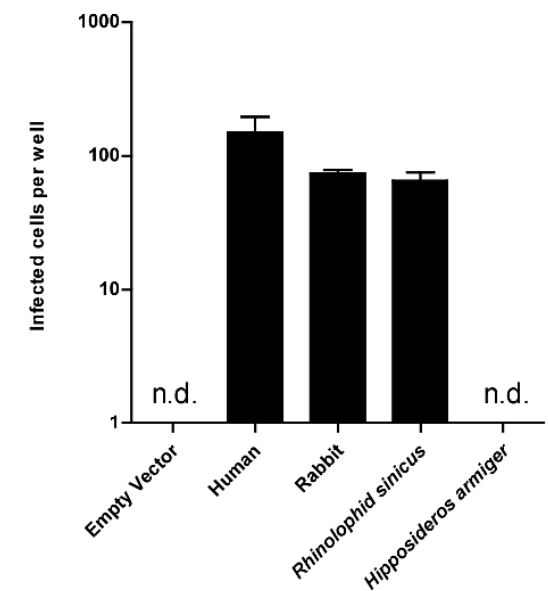

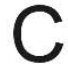
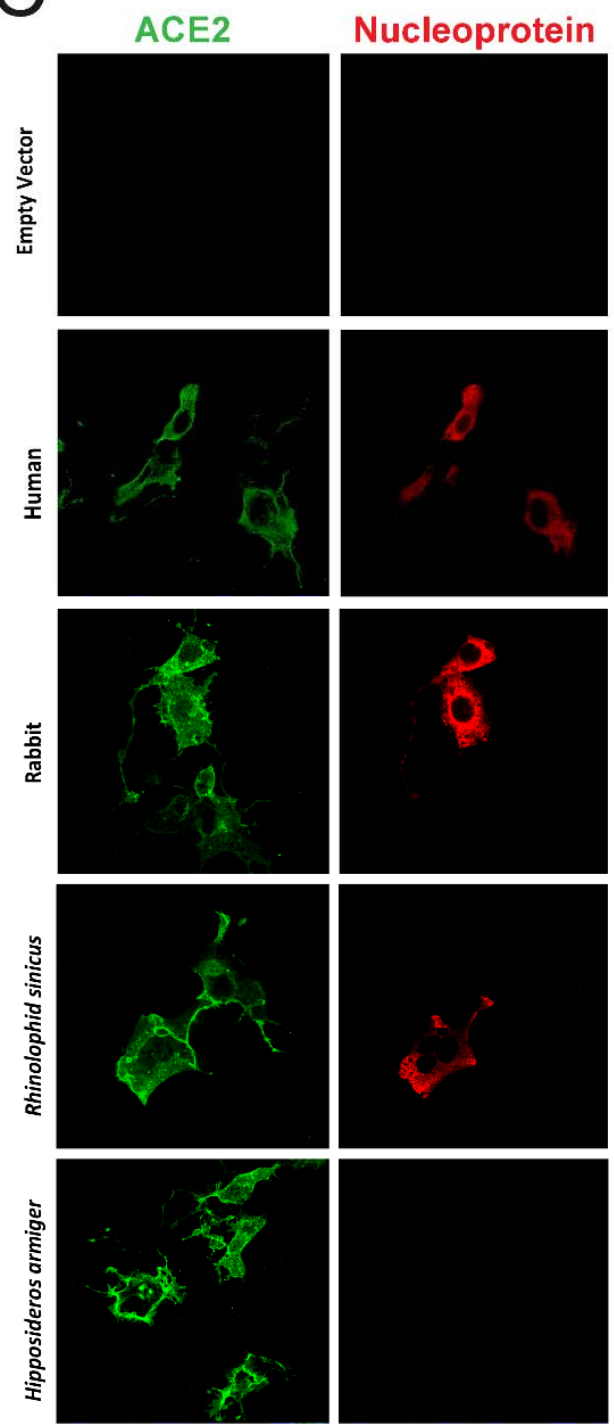

Merge
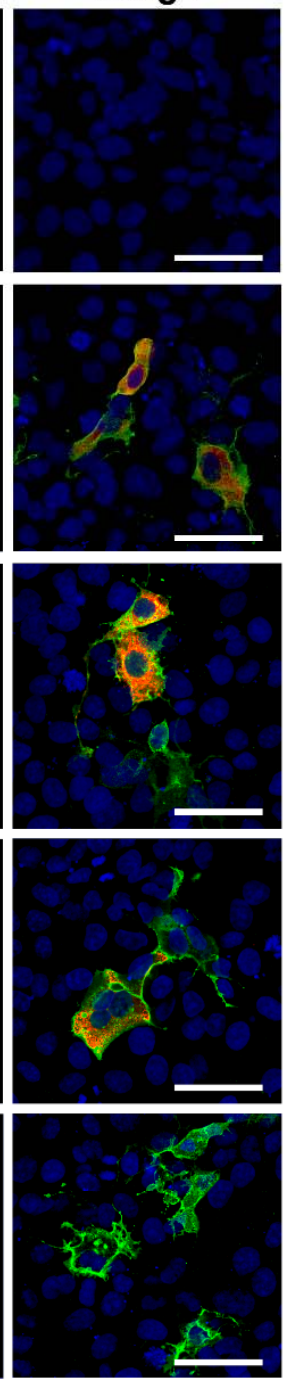

Figure 1. Rabbit ACE2 mediated SARS-CoV-2 infection. SARS-CoV-2 pseudovirus (A) and authentic virus (B) infection of Cos-7 cells expressing ACE2 of various species. Infectivity was quantified by staining live virus cells with anti-SARS-CoV nucleocapsid and scanning live virus and pseudovirus infected cells. (C) Confocal imaging of ACE2 mediated live virus infection; cells were stained using anti-human ACE2 in green, anti-SARS-CoV nucleocapsid in red and TO-PRO3 in blue to stain nuclei. Scale indicates $50 \mu \mathrm{m}$. 
bioRxiypreprint doi: https://doi.org/10.1101/2020.08.27.263988; this version posted August 27, 2020. The copyright holder for this preprint (which was not certified by peer review) is the author/funder, who has granted bioRxiv a license to display the preprint in perpetuity. It is made

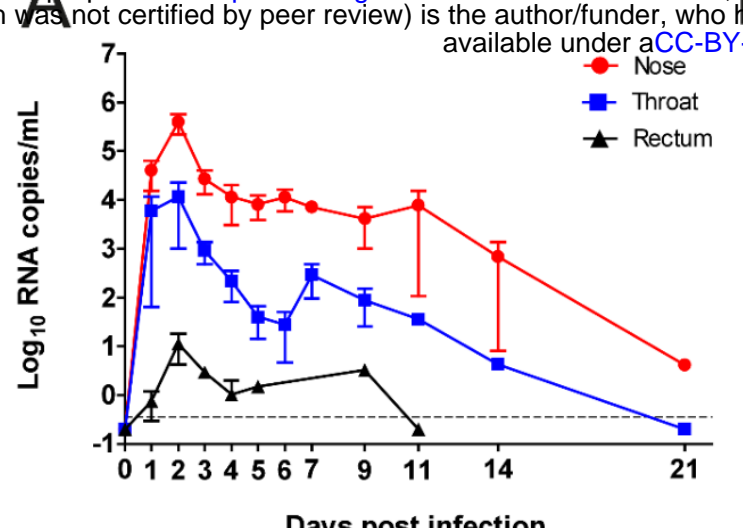

193

191

192

Days post infection
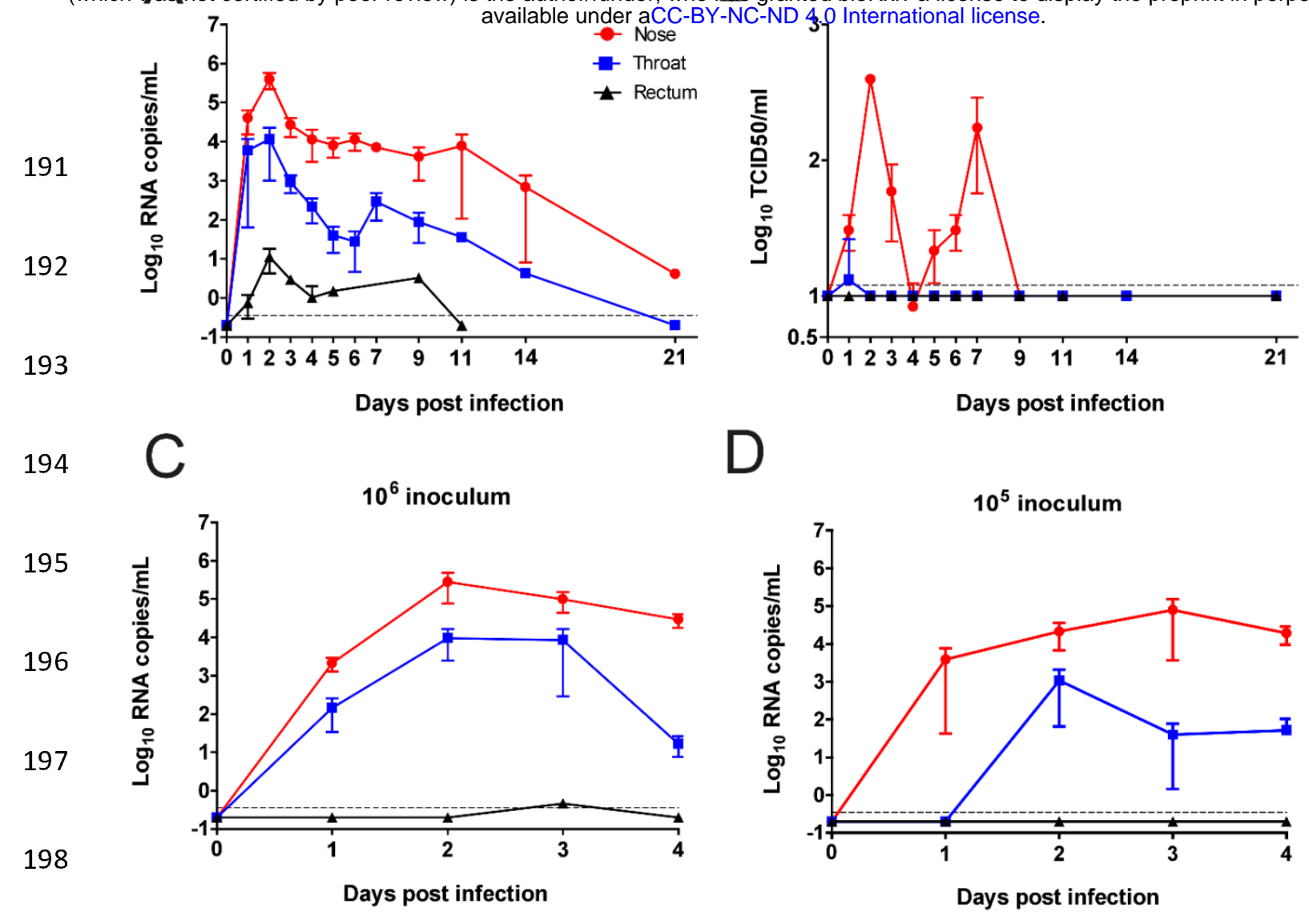

D
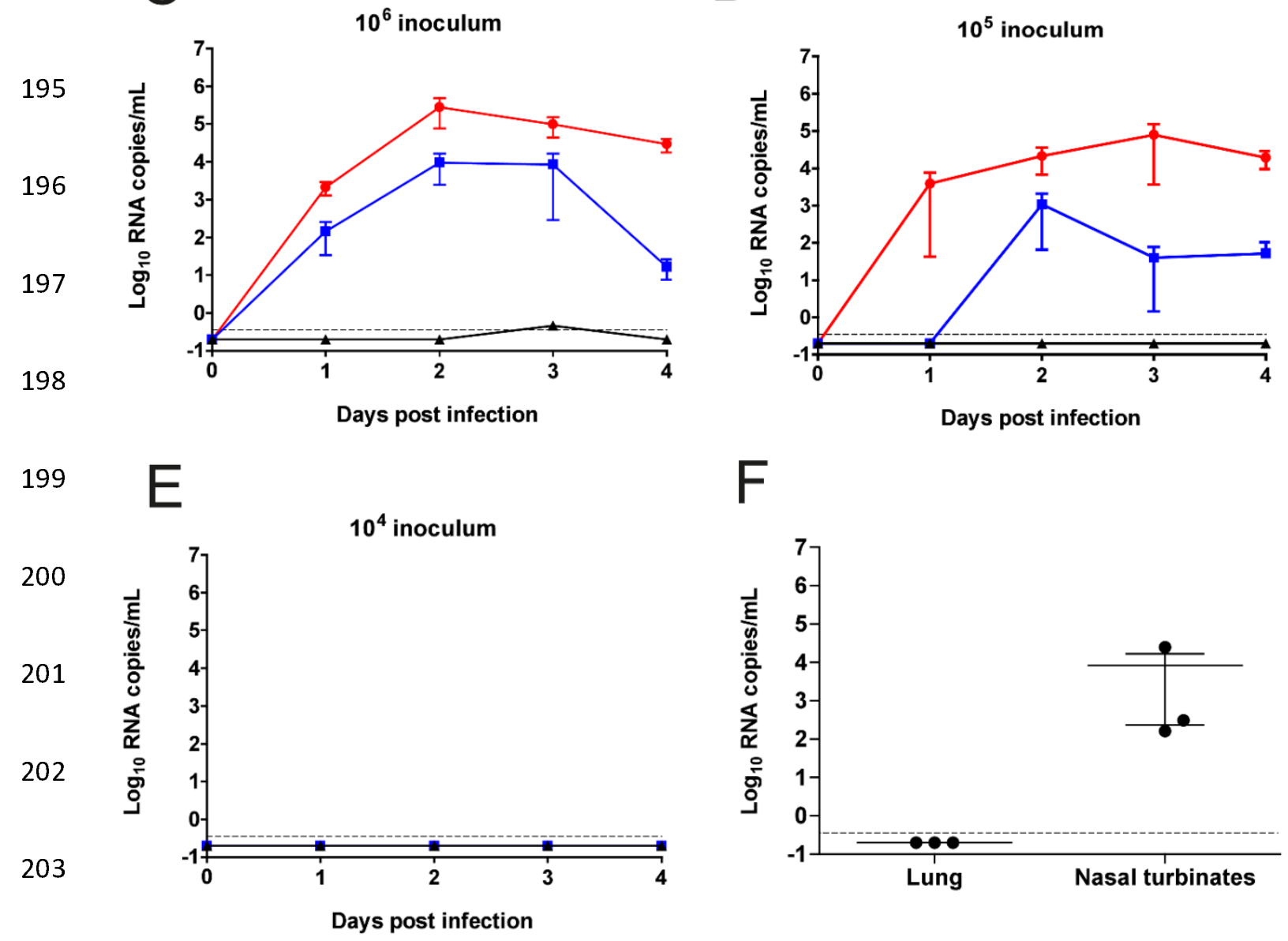

$\mathrm{F}$
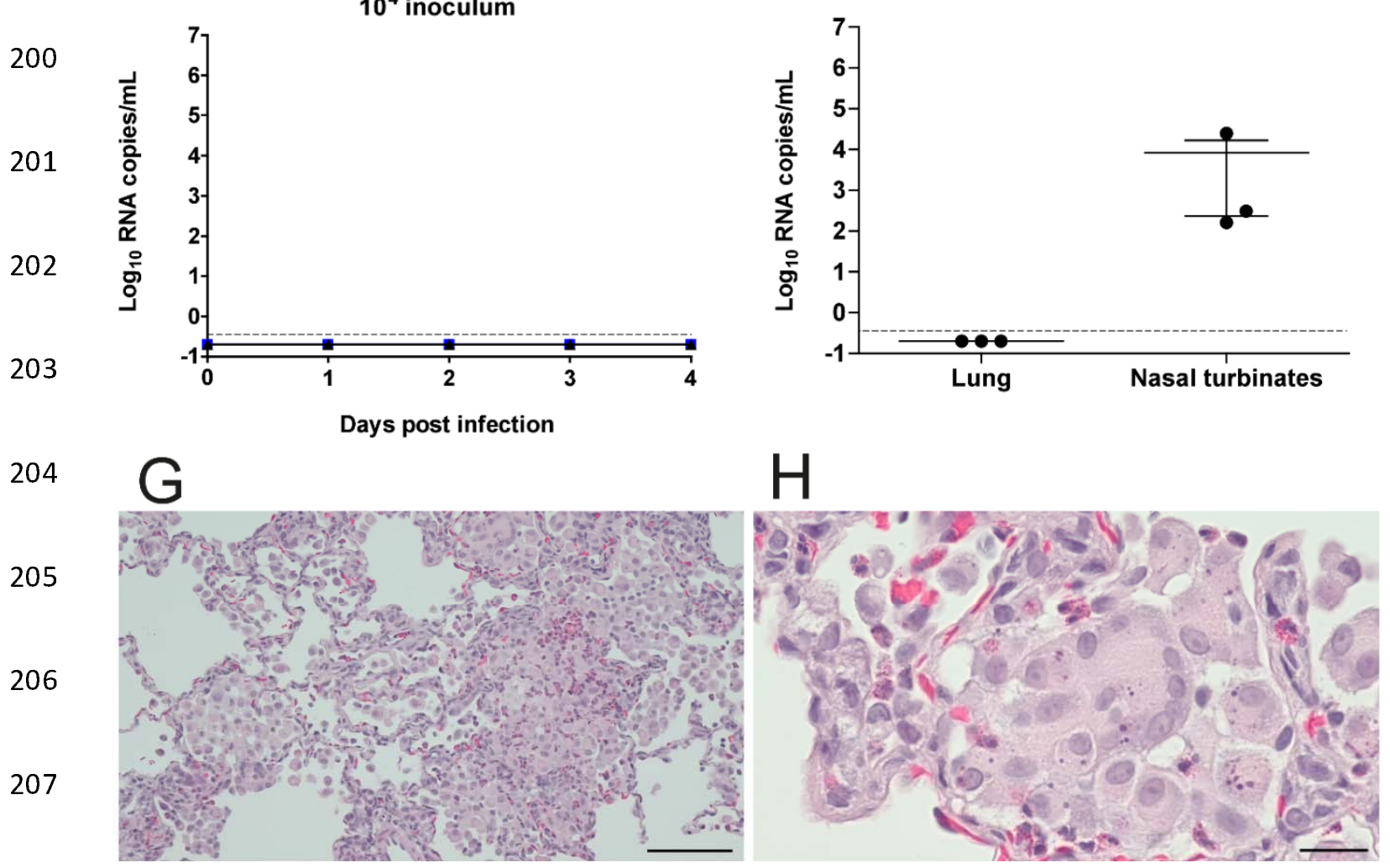

Figure 2. Susceptibility of rabbits to SARS-CoV-2 infection. Infection kinetics of (A) viral RNA and (B) authentic SARS-CoV-2 virus growth curves from rabbits inoculated with $10^{6} \mathrm{TCID}_{50}$ and followed up for 21 days. (C-E) Viral RNA growth curves in rabbits inoculated with either (C) $10^{6}$, (D) $10^{5}$, or (E) $10^{4} \mathrm{TCID}_{50}$ and followed up for four days post infection. (F) Viral RNA in lung and nasal turbinates of $10^{6}$ TCID $_{50}$ infected rabbits, sacrificed after four days. RNA detection limits were set at $3.5 \times 10^{-1}$ RNA copies $/ \mathrm{ml}$, while live virus detection limit was $12 \mathrm{TCID}_{50} / \mathrm{ml}$. (G, H) Histopathological analysis of lungs from rabbits inoculated with $10^{6} \mathrm{TCID}_{50}$, sacrificed after four days. (G) Alveolar thickening and inflammatory infiltrates. Scale indicates $100 \mu \mathrm{m}(\mathrm{H})$ Enlarged, 


\section{Supplementary}

Materials and Methods

\section{Expression plasmids and cloning}

212 Plasmids in pcDNA3.1 encoding human ACE2 (OHu20260), rabbit ACE2 (Clone ID

213 OOb21562D), Rhinolophus sinicus ACE2 (ORh96277) and Hipposideros armiger ACE2 (Clone

214 ID OHi02685) were ordered from GenScript. Codon-optimized cDNA encoding SARS-CoV-2 S

215 glycoprotein (isolate Wuhan-Hu-1) with a C-terminal 19 amino acid deletion was synthesized

216 and cloned into pCAGSS in between the EcoRI and BglII sites. pVSV-eGFP-dG (\#31842),

217 pMD2.G (\#12259), pCAG-VSV-P (\#64088), pCAG-VSV-L (\#64085), pCAG-VSV-N (\#64087)

218 and pCAGGS-T7Opt (\#65974) were ordered from Addgene. S expressing pCAGGS vectors

219 were used for the production of pseudoviruses, as described below.

220 Cell lines

221 HEK-293T cells were maintained in Dulbecco's Modified Eagle's Medium (DMEM, Gibco)

222 supplemented with $10 \%$ fetal bovine serum (FBS), $1 \mathrm{X}$ non-essential amino acids (Lonza), 1mM

223 sodium pyruvate (Gibco), 2mM L-glutamine (Lonza), $100 \mu \mathrm{g} / \mathrm{ml}$ streptomycin (Lonza) and 100

$224 \mathrm{U} / \mathrm{ml}$ penicillin. Cos-7, Vero, and VeroE6 cells were maintained in DMEM supplemented with

225 10\% FBS, $1.5 \mathrm{mg} / \mathrm{ml}$ sodium bicarbonate (Lonza), 10mM HEPES (Lonza), 2mM L-

226 glutamine, $100 \mu \mathrm{g} / \mathrm{ml}$ streptomycin and $100 \mathrm{U} / \mathrm{ml}$ penicillin. All cell lines were maintained at

$227 \quad 37^{\circ} \mathrm{C}$ in a $5 \% \mathrm{CO}_{2}$ humidified incubator. 
The protocol for VSV-G pseudovirus rescue was adapted from Whelan and colleagues (1).

Briefly, a 70\% confluent $10 \mathrm{~cm}$ dish of HEK-293T cells was transfected with $10 \mu \mathrm{g}$ pVSV-eGFP-

$\Delta \mathrm{G}, 2 \mu \mathrm{g}$ pCAG-VSV-N (nucleocapsid), $2 \mu \mathrm{g}$ pCAG-VSV-L (polymerase), $2 \mu \mathrm{g}$ pMD2.G

(glycoprotein, VSV-G), $2 \mu \mathrm{g}$ pCAG-VSV-P (phosphoprotein) and $2 \mu \mathrm{g}$ pCAGGS-T7Opt (T7

RNA polymerase) using polyethylenimine (PEI) at a ratio of 1:3 (DNA:PEI) in Opti-MEM I

$(1 X)+$ GlutaMAX. Forty-eight hours post-transfection the supernatant was transferred onto new plates transfected 24 hours prior with VSV-G. After a further 48 hours, these plates were retransfected with VSV-G. After 24 hours the resulting pseudoviruses were collected, cleared by centrifugation at $2000 \mathrm{x}$ g for 5 minutes, and stored at $-80^{\circ} \mathrm{C}$. Subsequently, VSV-G pseudovirus batches were produced by infecting VSV-G transfected HEK-293T cells with VSV-G pseudovirus at a MOI of 0.1 . Titres were determined by preparing 10 -fold serial dilutions in Opti-MEM I (1X) + GlutaMAX. Aliquots of each dilution were added to monolayers of $2 \times$ $10^{4}$ Vero cells in the same medium in a 96-well plate. Three replicates were performed per pseudovirus stock. Plates were incubated at $37^{\circ} \mathrm{C}$ overnight and then scanned using an Amersham Typhoon scanner (GE Healthcare). Individual infected cells were quantified using ImageQuant TL software (GE Healthcare). All pseudovirus work was performed in a Class II Biosafety Cabinet under BSL-2 conditions at Erasmus Medical Center.

\section{Coronavirus $\mathbf{S}$ pseudovirus production}

For the production of SARS-CoV-2 S pseudovirus, HEK-293T cells were transfected with $10 \mu \mathrm{g}$ S expression plasmids. Twenty-four hours post-transfection, the medium was replaced for OptiMEM I (1X) + GlutaMAX, and cells were infected at an MOI of 1 with VSV-G pseudovirus. Two hours post-infection, cells were washed three times with OptiMEM and replaced with medium containing anti-VSV-G neutralizing antibody (clone 8G5F11; Absolute Antibody) at a 
252 dilution of 1:50,000 to block remaining VSV-G pseudovirus. The supernatant was collected after

25324 hours, cleared by centrifugation at $2000 \mathrm{x}$ g for 5 minutes and stored at $4{ }^{\circ} \mathrm{C}$ until use within 7

254 days. SARS-CoV-2 pseudovirus was titrated on VeroE6 cells as described above.

Virus stock

256

257

258

259

260

261

262

263

264

265

266

267

268

269

270

271

272

273

SARS-CoV-2 (isolate BetaCoV/Munich/BavPat1/2020; European Virus Archive Global \#026V03883; kindly provided by Dr. C. Drosten) was propagated on Vero E6 (ATCC® CRL 1586 ${ }^{\mathrm{TM}}$ ) cells in OptiMEM I (1X) + GlutaMAX (Gibco), supplemented with penicillin (100 IU/mL) and streptomycin $(100 \mathrm{IU} / \mathrm{mL})$ at $37^{\circ} \mathrm{C}$ in a humidified $\mathrm{CO} 2$ incubator. Stocks were produced by infecting Vero E6 cells at a multiplicity of infection (MOI) of 0.01 and incubating the cells for 72 hours. The culture supernatant was cleared by centrifugation and stored in aliquots at $-80^{\circ} \mathrm{C}$.

Stock titers were determined by titratin on VeroE6 cells. The TCID $_{50}$ was calculated according to the method of Spearman \& Kärber (ref?). All work with infectious SARS-CoV-2 was performed in a Class II Biosafety Cabinet under BSL-3 conditions at Erasmus Medical Center.

\section{Pseudovirus and live virus infection in vitro}

Cos-7 cells plated at $70 \%$ density in a 24 well format were transfected 24 hours after plating by dropwise addition of $500 \mathrm{ng}$ ACE2 expression plasmids using a PEI ratio of 1:3 (DNA:PEI) in

Opti-MEM I (1X) + GlutaMAX. After 24 hours, cells were washed twice and replaced with fresh Opti-MEM I (1X) + GlutaMAX prior to pseudovirus or live virus infection. Pseudovirus transduction was performed by infecting plates with $10^{3}$ VeroE6 titrated particles per well. Plates were incubated for 16 hours at $37^{\circ} \mathrm{C}$ before quantifying GFP-positive cells using an Amersham Typhoon scanner and ImageQuant TL software. Authentic virus infection was performed by adding $10^{4}$ TCID $_{50}$ SARS-CoV-2 per well and incubating plates for 8 hours at $37^{\circ} \mathrm{C}$. After 
274 incubation, cells were formalin fixed, permeabilized with 70\% ethanol and stained with 1:1000

275 mouse anti-SARS nucleoprotein (Sino Biological) and 1:1000 rabbit anti-human ACE2

276 (Abcam), followed by 1:1000 goat anti-rabbit Alexa-Fluor 594, 1:1000 goat anti-mouse Alexa-

277 Fluor 488, and 1:1000 TO-PRO3 (Thermo Fisher) to stain nuclei. Quantification of virus

278 infected cells was performed using an Amersham Typhoon scanner and ImageQuant TL software

279 as described above, while confocal imaging was performed on a LSM700 confocal microscope

280 using ZEN software (Zeiss).

\section{In vivo study design}

Animal experiments were approved and performed according to the guidelines from the

Institutional Animal Welfare Committee (AVD277002015283-WP04). The studies were

SARS-CoV-2 and monitored for 21 days post infection. Animals monitored for four days were infection. In addition, on day 21 sera was collected from these animals for serological testing. 
degrees Celsius for 30 minutes) samples were 2-fold serially diluted in Dulbecco modified Eagle medium supplemented with NaHCO3, HEPES buffer, penicillin, streptomycin, and $1 \%$ fetal bovine serum, starting at a dilution of $1: 10$ in $50 \mu \mathrm{L}$. Fifty $\mu \mathrm{L}$ virus suspension ( 400 plaqueforming units) was added to each well and incubated at $37^{\circ} \mathrm{C}$ for 1 hour before transferring to an 8 hour incubation. After incubation, the cells were fixed with $4 \%$ formaldehyde/phosphatebuffered saline (PBS) and stained with polyclonal rabbit anti-SARS-CoV antibody (Sino Biological), followed by a secondary peroxidase-labeled goat anti-rabbit IgG (Dako). The signal was developed by using a precipitate forming 3,3',5,5' tetramethylbenzidine substrate (True quantified using an ImmunoSpot Image Analyzer (CTL Europe GmbH). The serum neutralization titer is the reciprocal of the highest dilution resulting in an infection reduction of $>50 \%\left(\mathrm{PRNT}_{50}\right)$. A titer $>20$ was considered positive.

\section{RNA extraction and qRT-PCR}

311 Swabs, lung homogenates and nasal turbinate homogenates were thawed and centrifuged at

$3122,000 \mathrm{x}$ g for $5 \mathrm{~min}$. Sixty $\mu \mathrm{L}$ supernatant was lysed in $90 \mu \mathrm{L}$ MagnaPure LC Lysis buffer

313 (Roche) at room temperature for 10 minutes. RNA was extracted by incubating samples with 50

$314 \mu \mathrm{L}$ Agencourt AMPure XP beads (Beckman Coulter) for 15 minutes at room temperature,

315 washing beads twice with 70\% ethanol on a DynaMag-96 magnet (Invitrogen) and eluting in 30

$316 \mu \mathrm{L}$ ultrapure water. RNA copies per $\mathrm{mL}$ were determined by qRT-PCR using primers targeting

317 the E gene (37) and compared to a counted RNA standard curve. 
Alveolitis severity, bronchitis/bronchiolitis severity, tracheitis and rhinitis severity were scored:

$0=$ no inflammatory cells, $1=$ few inflammatory cells, $2=$ moderate number of inflammatory

321 cells, $3=$ many inflammatory cells. Alveolitis extent, $0=0 \%, 1=<25 \%, 2=25-50 \%, 3=>50 \%$.

322 Alveolar oedema presence, alveolar haemorrhage presence, type II pneumocyte hyperplasia

323 presence, $0=$ no, $1=$ yes. Extent of peribronchial/perivascular cuffing, $0=$ none, $1=1-2$ cells

324 thick, $2=3-10$ cells thick, $3=>10$ cells thick.

\section{Immunohistochemistry}

326 Semiquantitative assessment of SARS-CoV-2 viral antigen expression in the lungs was

327 performed as reported for SARS-CoV earlier (3) with a few amendments: for the alveoli, twenty-

328 five arbitrarily chosen, 20x objective fields of lung parenchyma in each lung section were

329 examined by light microscopy for the presence of SARS-CoV-2 nucleoprotein, without the

330 knowledge of the allocation of the animals. The cumulative scores for each animal were

331 presented as number of positive fields per 100 fields. For the bronchi and bronchioles, the

332 percentage of positively staining bronchial and bronchiolar epithelium was estimated on every

333 slide and the average of the four slides was taken to provide the score per animal. For the trachea

334 and nose, the percentage of positively staining epithelium was estimated on every slide.

\section{References}

336 1. Whelan SP, Ball LA, Barr JN, Wertz GT. Efficient recovery of infectious vesicular

337 stomatitis virus entirely from cDNA clones. Proc Natl Acad Sci U S A. 1995 Aug

$338 \quad 29 ; 92(18): 8388-92$. 
2. Okba NMA, Muller MA, Li W, Wang C, GeurtsvanKessel CH, Corman VM, et al.

340 Severe Acute Respiratory Syndrome Coronavirus 2-Specific Antibody Responses in Coronavirus

341 Disease Patients. Emerg Infect Dis. 2020 Jul;26(7):1478-88.

342

344

345

3. Haagmans BL, Kuiken T, Martina BE, Fouchier RA, Rimmelzwaan GF, van Amerongen

G, et al. Pegylated interferon-alpha protects type 1 pneumocytes against SARS coronavirus infection in macaques. Nat Med. 2004 Mar;10(3):290-3.

\section{Supplementary Figures}
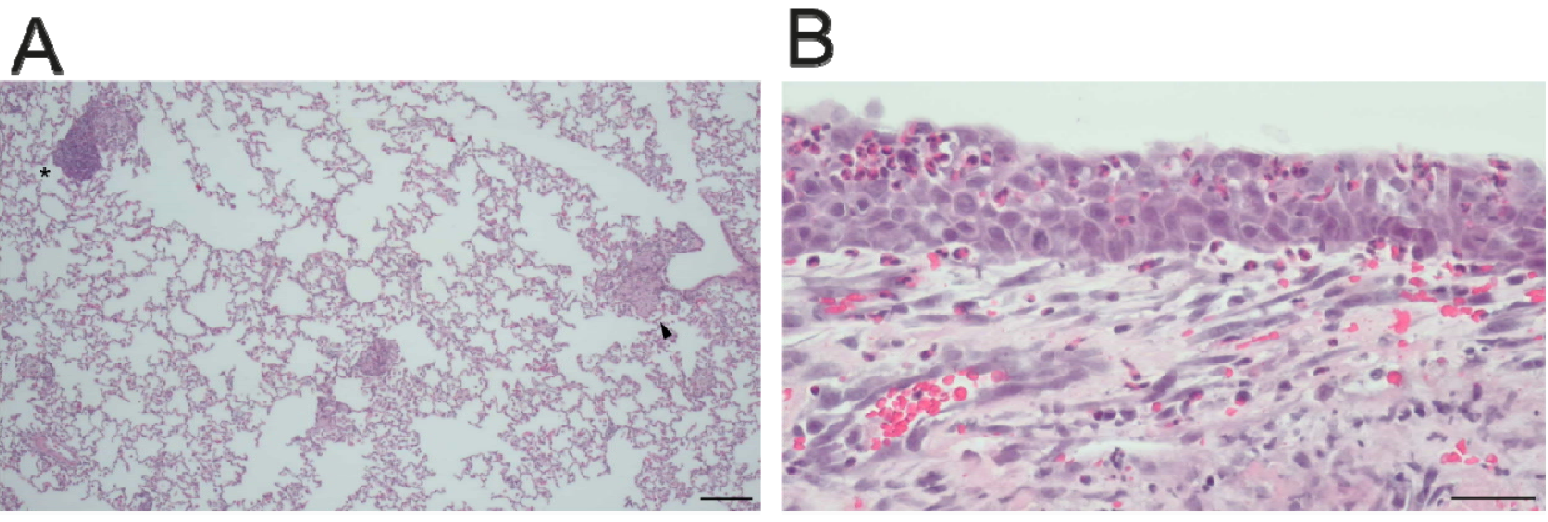

Supplementary Figure 1. Lung histology of SARS-CoV-2 infected rabbits. (A) Lung pathology overview from rabbits inoculated with $10^{6} \mathrm{TCID}_{50}$ and sacrificed 4 days post infection. Arrow indicates thickening and asterisk bronchus-associated lymphoid tissue (BALT). Scale indicates $200 \mu \mathrm{m}$. (B) Eosinophilic infiltrates in the nose. Scale indicates $40 \mu \mathrm{m}$. 